\title{
Tear biomarkers in neurodegenerative diseases
}

\author{
STANESCU Ioana ${ }^{1}$, BULBOACĂ Adriana ${ }^{2}$, DOGARU Gabriela ${ }^{3}$, NICULA Cristina ${ }^{4}$ \\ Corresponding author: Adriana E Bulboaca, E-mail: adriana bulboaca@yahoo.com
}

1 Department of Neuroscience, "Iuliu Hatieganu" University of Medicine and Pharmacy, Cluj Napoca, Romania 2 Department of Pathophysiology, "Iuliu Hatieganu" University of Medicine and Pharmacy, Cluj Napoca, Romania 3 Department of Physical Medicine and Medical Rehabilitation, "Iuliu Hatieganu" University of Medicine and Pharmacy,

Cluj Napoca, Romania

4 Department of Ophthalmology, "Iuliu Hatieganu” University of Medicine and Pharmacy, Cluj Napoca, Romania

\begin{abstract}
In neurodegenerative diseases (NDD) early and accurate diagnosis is a priority in neurological research, as treatment is most effective when started early in the disease evolution. Biomarkers could be used as an assessment tool for high risk population, for monitoring disease progression and evaluation of treatment efficacy. The close connection between the eye and the brain leads to the hypothesis of detecting biomarkers for neurological diseases in tear fluid, collected by non-invasive methods and with a relatively stable composition.

A combination of four tear proteins (lipocalin-1, lysozyme-C, lacritin and dermcidin) is demonstrated to be specific for Alzheimer's disease (AD). Asymptomatic patients which showed increased volume and protein content of tear secretion, and decreased levels of lipocalin-1, lysozyme-C and lacritin and increased levels of dermcidin, may be considered at risk of developing $\mathrm{AD}$, and may be subjects to further psychological and neuroimagistic tests.

In Parkinson's disease (PD), tear levels of oligomeric a-synuclein are considered to be a strong biomarker. Also, high tear levels of oligomeric a-synuclein could precede the onset of clinical manifestations of PD, with possible utility as screening tool in asymptomatic people. The diagnosis of NDD through the eye window could be an important step forward in personalized medicine.
\end{abstract}

Key words: neurodegenerative diseases, fluid biomarkers, tear composition,

\section{Introduction}

Neurodegenerative diseases (NDD) are a group of age-dependent, chronic disorders characterized by progressive dysfunction and loss of neurons, which became increasingly prevalent. Pathogenesis is related to deposition of proteins with altered physicochemical properties in brain cells (misfolded proteins), followed by the involvement of different functional systems in the nervous system, with specific clinical presentation (1).

Early and accurate diagnosis of NDD before the occurrence of clinical symptoms is a priority in neurological research, as treatment is most effective when started early in the disease evolution. Usually, the methods currently available have less applicability for the screening of general population, and there is a high demand for novel diagnostic methods using non-invasive and accessible tools for detecting NDD in their pre-clinical stage or groups of high-risk individuals.

Biomarkers are a useful tool in detecting the risk of developing a disease, in assessing the rate of disease progression and activity, and in predicting the response to a specific therapy (2). A biomarker is a "defined characteristic that is measured as an indicator of normal biological processes, pathogenic processes, or responses to an exposure or intervention, including therapeutic interventions" (3).

Biomarker levels in biofluids (serum, plasma, cerebrospinal fluid or tears) have been measured in several NDD, with the aim of finding a diagnostic or prognostic parameter (4). Biomarkers collected by noninvasive methods are valuable, especially for screening high-risk populations.

Tears are an accessible body fluid, with relatively simple composition and non-invasive sample collection. There are four types of tears: basal, reflex, emotional and closed eye. Samples are collected through Schirmer strips, minisponges, microcapillary tubes or eye-wash, without inducing reflex tearing, and should be stored in standard conditions $(5,6)$. 
The lacrimal glands are part of the "lacrimal functional unit", together with the ocular surface (cornea, conjunctiva and meibomian glands) and the interconnected sensory and motor nerve fibers (7). The volume and the composition of the tear film is controlled by this unit, and consequently, the secretion of specific tear proteins is influenced by changes in neuronal function. The lacrimal glands are innervated by autonomic sympathetic and parasympathetic nerves; the cholinergic nerves stimulates the tear secretion. The flow rate of tears is thus influenced by autonomic pathways, and is highly sensitive to autonomic dysfunction $(8,9,10)$. Tear functions consist in lubrication of the eyelids during blinking, maintaining homeostasis of eye surface and preservation of the corneal surface (11). The tear film creates a chemical and immunological barrier at eye's surface, protecting against physical and biological agents (12).

\section{Tear biomarkers in clinical practice}

Tear film is formed by a muco-aqueous layer, which contains proteins, and a lipidic layer (11). Tears contain a mixture of proteins, peptides (including between 1500 and 2000 types identified by proteomics), lipids (6 classes with 150 species), mucins, small molecule metabolites (100 types identified), nucleic acids (microRNAs), electrolytes and water, secreted from lacrimal glands, Meibomian glands, goblet cells and ocular surface epithelial and nerve cells $(2,10,13,14,15)$. The assessment of tear composition represents an ideal tool for biomarker detection.

Tear protein content is high, between $6-10 \mathrm{mg} / \mathrm{ml}$, and the major tear proteins, such as lipocalin-1, lactotransferrin and lysozyme-C, are involved in local immunologic and inflammatory processes $(9,16)$. Two thirds of the proteins are tear-specific, and one third is common with plasma proteins (10).

Multiple tear biomarkers have been described in ocular diseases such as: dry eye disease (DED), diabetic retinopathy, glaucoma, giant papillary conjunctivitis, vernal kerato-conjunctivitis, peripheral ulcerative keratitis, Sjorgren's syndrome (SS), thyroid-associated orbitopathy (TAO), etc. In this group, useful biomarkers detected were: lactoferrin, matrix-metalloproteinase-9 (MMP-9) for DED, inflammatory cytokines (interleukin-8, interferon-gamma, tumor necrosis factor-alpha, interleukin-1, interleukin-6, $-17,-22$, etc) and chemokines - for DED, TAO and SS $(2,17)$.
Tear composition is a source of biomarkers in systemic diseases, such as: diabetes, systemic sclerosis, cystic fibrosis, rheumatoid arthritis and cancer $(2,10)$. Lacryglobin was shown to be secreted in higher amounts in the tears of patients with colon, prostate, breast and lung cancers (18).

\section{Tear biomarkers in neurodegenerative diseases}

The close connection between the eye and the brain leads to the hypothesis of detecting specific ocular biomarkers for neurological diseases (2). Biomarkers in NDD could be used as risk assessment in general population, monitoring of disease progression and evaluation if treatment efficacy. The eye shares neural similarities with the brain, and is considered to be a target in the research field of biomarkers in NDD (19).

For the anterior pole, tear fluid is one of the most important source of biomarkers, as changes in tear composition have already been described in systemic and neurological diseases. Lacrimal glands are highly innervated by cholinergic neurons, and influenced by adrenergic and dopaminergic pathways, and characteristic tear protein profiles could be detected if alterations of specific neural pathways occurred $(20,21)$.

In two of the most frequent neurodegenerative diseases - Alzheimer's dementia (AD) and Parkinson's disease (PD) - visual impairments has already been described (22). Oxidative stress and inflammatory mechanisms could represent a common pathogenic chain in degenerative ophthalmologic and neurologic diseases, but tear abnormalities could be also linked to associated dysfunctions in autonomic nervous system. NDD are also associated with altered tear production and decrease corneal sensitivity (23).

The ocular involvement in $\mathrm{AD}$ affects the anterior and the posterior pole of the eye. Maintaining the integrity of visual pathways should be an important objective in assessing NDD evolution and prognosis (24). Neurodegenerative changes detected in the brain of an $\mathrm{AD}$ patient are presumably associated with structural changes of the neuroretina (19). Damage of the retinal neurons have been described in $\mathrm{AD}$, ganglionic cell loss and optic nerve degeneration could lead to visual impairment $(25,26$, 27). Beta-amyloid plaques were found in the retina of $\mathrm{AD}$ patients from an early stage of the disease (28). 
Also, beta-amyloid deposits were found in the eyes of patients with glaucoma and age-related macular degeneration, suggesting a link with $\mathrm{AD}$ pathogenesis $(29,30)$. Alteration in retinal structure and blood flow impair the eye microenvironment in $\mathrm{AD}$, leading to anterior pole involvement and changes in tear composition.

Lacrimal gland dysfunction has been demonstrated in $\mathrm{AD}$, showing increase tear flow rate and increased protein content (9). Proteins that are differently expressed in tears of patients with $\mathrm{AD}$ are those involved in local defense mechanisms and eye chemical barrier, so changes in tear composition may involve a greater risk for ocular infection (9). In the tears of patients suffering from $\mathrm{AD}$, lower levels of lipocalin-1, lactotransferrin, lysozyme-C, extracellular glycoprotein lacritin and prolactininducible protein and higher levels of dermcidin were described (9). Also, elevated levels of nucleic acid - microRNA-200b-5p were detected in the tear samples of patients with AD, compared to controls (16).

Based on these findings, a combination of four tear proteins could be used as a screening tool for $\mathrm{AD}$ diagnosis in asymptomatic patients: lipocalin-1, lysozyme-C, lacritin and dermcidin. Asymptomatic patients which shows a combination of these biomarkers with increased tear volume and protein content, may be considered at risk of developing $\mathrm{AD}$, and may be subjects to further psychological and neuroimagistic tests. These tear markers are more valuable as actually there is no tool for population screening in $\mathrm{AD}$.

Visual dysfunction has already been described in PD patients, being caused by dopaminergic depletion and accumulation of a pathologic protein, alphasynuclein, in the retina $(31,32)$.

Tear secretion in PD patients is influenced by the specific dysfunction of autonomic nervous system. In a study, the PD patients have a decreased blink rate and tear secretion, compared to controls, complaining of dry eye sensation and blepharitis (33). The dysfunction of cholinergic pathways in PD patients influence lacrimal gland secretion, and is involved in changing tear composition.

The tears of patients with Parkinson's disease (PD) contain biomarkers that can be directly tested. High levels of tumor necrosis factor alpha (TNF-a), DJ-1 protein (a cysteine protease encoded by PARK7 gene) (34), oligomeric alpha-synuclein (a-syn), and decreased levels of total a-syn have been described in tear fluid of parkinsonian patients $(8,21)$.
Alpha-synuclein is the main abnormal protein identified in the brain of PD patients, being one of the components of Lewy bodies (35). Neurons which innervates lacrimal glands of PD patients are affected by neurodegenerative changes, and may release oligomeric a-synuclein into the tear fluid. On the other hand, rich corneal sensory innervation could be another source of oligomeric a-synuclein from ciliary nerves terminations (10).

Oligomeric a-syn is considered to be a strong biomarker for PD, especially in male patients. DJ-1 protein was also increased in the tears of male PD patients compared to controls (21).

It has been hypothesized that abnormal high levels of oligomeric a-syn in tears and other biofluids could precede the onset of clinical manifestations of PD, and could be used at a screening tool in detecting the risk of disease in asymptomatic people (21).

In amyotrophic lateral sclerosis (ALS), a severe NDD in which central and peripheral motoneurons are affected, an alteration of corneal small sensory fibers has been described, but there is no study regarding tear composition (36).

In Hungtington disease, there are no clinicaly validated biofluid markers available for screening or outcome (37).

\section{Conclusions}

Tear analysis is an uncomplicated and easy to perform test, that can be used in the screening process of the general population for degenerative diseases. There are some evidences that tear biomarkers could predict with specificity the risk for NDD, and patients with positive tests may be further evaluated by specialists (9). As tears are the most important source of eye biomarkers, there is a strong reason for the development and validation of new and precise non-invasive tools to facilitate the early detection of neurodegenerative diseases and the prediction of their progression. Further research are needed to increase the specificity of tests and of tear biomarkers panel (38). 


\section{References}

1. Kovacs GG. Concepts and classification of neurodegenerative diseases. In Handbook of Clinical Neurology, Vol. 145 (3rd series); Neuropathology, edited by G.G. Kovacs and I. Alafuzoff, Elsevies. 2017; 21: 301-306.

2. Hagan S, Martin E, Enríquez-de-Salamanca A. Tear fluid biomarkers in ocular and systemic disease: potential use for predictive, preventive and personalised medicine. EPMA J. 2016;7(1):15. Published 2016 Jul 13.

3. BEST (Biomarkers, EndpointS, and other Tools) Resource: FDA-NIH Biomarker working Group; 2018. $61 \mathrm{p}$

4. Pujol-Lereis LM. Alteration of Sphingolipids in Biofluids: Implications for Neurodegenerative Diseases. Int J Mol Sci. 2019 Jul 21;20(14):3564. doi: 10.3390/ijms20143564. PMID: 31330872 ; PMCID: PMC6678458.

5. Fullard RJ, Snyder C. Protein levels in nonstimulated and stimulated tears of normal human subjects. Invest Ophthalmol Vis Sci.1990;31(6):1119-26

6. Fullard RJ, Tucker D. Tear protein composition and the effects of stimulus. Adv Exp Med Biol. 1994;350:309-14.

7. Stern ME, Beuerman RW, Fox RI, Gao J, Mircheff AK, Pflugfelder SC. The pathology of dry eye: the interaction between the ocular surface and lacrimal glands. Cornea. 1998;17(6):584-9

8. Feigenbaum D, Lew M, Janga S, Shah MK, Mack W, Okamoto C, Hamm-Alvarez S. Tear Proteins as Possible Biomarkers for Parkinson's Disease, Neurology Apr 2018, 90 (15 Supplement) S3.006

9. Kalló G, Emri M, Varga Z, Ujhelyi B, Tőzsér J, Csutak A, Csősz É. Changes in the Chemical Barrier Composition of Tears in Alzheimer's Disease Reveal Potential Tear Diagnostic Biomarkers. PLoS One. 2016 Jun 21;11(6): 0158000.

10.1371/journal.pone.0158000. doi: 27327445; PMCID: PMC4915678.

10. Edman MC, Janga SR, Kakan SS, et al. Tears more to them than meets the eye: why tears are a good source of biomarkers in Parkinson's disease. Biomark Med. 2020;14(2):151-163. doi:10.2217/bmm-2019-0364
11. Tamhane, M., Cabrera-Ghayouri, S., Abelian, G. et al. Review of Biomarkers in Ocular Matrices: Challenges and Opportunities. Pharm Res 36, 40 https://doi.org/10.1007/s11095-019-2569-8

12. Sperandio B, Fischer N, Sansonetti PJ. Mucosal physical and chemical innate barriers: Lessons from microbial evasion strategies. Semin Immunol. Elsevier Ltd; 2015; 27: 111-118

13. Zhou L, Beuerman RW. Tear analysis in ocular surface diseases. Prog Retin Eye Res. 2012;31(6):527-50.

14. Aass C, Norheim I, Eriksen EF, Thorsby PM, Pepaj M. Single unit filter-aided method for fast proteomic analysis of tear fluid. Anal Biochem. 2015;480:1-5. doi: 10.1016/j.ab.2015.04.002.

15. Semba RD, Enghild JJ, Venkatraman V, Dyrlund TF, Van Eyk JE. The human eye proteome project: perspectives on an emerging proteome. Proteomics. 2013;13(16):2500-11.

16. Kenny A, Jiménez-Mateos EM, Zea-Sevilla MA, Rábano A, Gili-Manzanaro P, Prehn JHM, Henshall DC, Ávila J, Engel T, Hernández F. Proteins and microRNAs are differentially expressed in tear fluid from patients with Alzheimer's disease. Sci Rep. 2019 Oct 28;9(1):15437. doi: 10.1038/s41598-019-51837y. PMID: 31659197 ; PMCID: PMC6817868.

17. Ionescu IC, Corbu CG, Tanase C, Ionita G, Nicula C, Coviltir V, Potop V, Constantin M, Codrici E, Mihai S, Popescu ID, Enciu A, Dascalescu D, Burcel M, Ciuvulica R, Voinea LM. Overexpression of Tear Inflammmatory Cytokines as additional Findings in Keratoconus Patients and Their First-Degree Family Members. Hindawi, Mediators of Inflammation ,2018,(2018), 4285268.

18. Evans V, Vockler C, Friedlander M, Walsh B, Willcox MD. Lacryglobin in human tears, a potential marker for cancer. Clin Experiment Ophthalmol. 2001;29(3):161-3.

19. Colligris P, Perez de Lara MJ, Colligris B, Pintor J. Ocular Manifestations of Alzheimer's and Other Neurodegenerative Diseases: The Prospect of the Eye as a Tool for the Early Diagnosis of Alzheimer's Disease. $J$ Ophthalmol. 2018;2018:8538573. Published 2018 Jul 30. doi:10.1155/2018/8538573 
20. Dartt DA. Neural regulation of lacrimal gland secretory processes: relevance in dry eye disease. Prog. Retin.Eye Res. 2009;28(3):155177.

21. Hamm-Alvarez SF, Okamoto CT, Janga SR, Feigenbaum D, Edman MC, Freire D, Shah M, Ghanshani R, Mack WJ, Lew MF. Oligomeric a-synuclein is increased in basal tears of Parkinson's patients. Biomark. Med. 2019;13(11):941-952

22. Johnson IP. Age-related neurodegenerative disease research needs aging models. Front Aging Neurosci. 2015;7:168.

23. Örnek N, Dag E, Örnek K. Corneal Sensitivity and Tear Function in Neurodegenerative Diseases. Current Eye Research. 2014:1-6

24. Bulboacã AE, Bulboacã A, Stanescu I, Blidaru M, Bulboacã AI, Cristina Nicula C, Dogaru G, Post-stroke visual rehabilitation - the impact on life quality of the stroke survival patients - a brief review. Balneo Research Journal. 2019;10(1):17-23.

25. La Morgia C, Ross-Cisneros FN, Koronyo Y, et al. Melanopsin retinal ganglion cell loss in Alzheimer disease. Ann Neurol. 2016;79(1): 90109.

26. Koronyo Y, Biggs E, Barron E et al. Retinal amyloid pathology and proof-of-concept imaging trial in Alzheimer's disease. JCI Insight. 2017;2(16)

27. Iseri $\mathrm{PK}$, Altinas $\mathrm{T}$, Tokay $\mathrm{T}$, Yuksel $\mathrm{N}$. Relationship between cognitive impairment and retinal morphological and visual functional abnormalities in Alzheimer disease. Journal of Neuro-Ophthalmology.2006:26(1):18-24.

28. Jiang J, Wang H, Li W, Cao X, Li C. Amyloid plaques in retina for diagnosis in Alzheimer's patients: a metaanalysis. Frontiers in Aging Neuroscience. 2016;8:267.

29. Johnson LV, Leitner WP, Rivest AJ, Staples MK, Radeke MJ, Anderson DH. The Alzheimer's a betapeptide is deposited at sites of complement activation in pathologic deposits associated with aging and age-related macular degeneration. Proceedings of the National Academy of Sciences of the United States of America. 2002; 99(18): 11830-11835

30. Mancino R, Martucci M, Cesareo $M$ et al. Glaucoma and Alzheimer disease: a single agerelated neurodegenerative disease of the brain. Current Neuropharmacology. 2017;16(7): 971977
31. Turcano P, Chen JJ, Savica R, et al. Early ophthalmologic features of Parkinson's disease: A review of preceding clinical and diagnostic markers. J Neurol. 2019;266:2103-2111.

32. Veys L, Vandenabeele M, Ortuño-Lizarán I, et al. Retinal a-synuclein deposits in Parkinson's disease patients and animal models. Acta Neuropathol. 2019;137:379-395.

33. Biousse V, Skibell BC, Watts RL, Loupe DN, Drews-Botsch C, Newman NJ. Ophthalmologic features of Parkinson's disease. Neurology.2004; 62(2):177-180.

34. Ariga H, Takahashi-Niki K, Kato I, Maita H, Niki T, Iguchi-Ariga SM. Neuroprotective function of DJ-1 in Parkinson's disease. Oxid Med Cell Longev. 2013;2013:683920

35. Goedert M. NEURODEGENERATION. Alzheimer's and Parkinson's diseases: The prion concept in relation to assembled $\mathrm{A} \beta$, tau, and $\alpha$ synuclein. Science. $\quad 2015 \quad$ Aug 7;349(6248):1255555

36. Ferrari G, Grisan E, Riva N, et al. Corneal confocal microscopy reveals trigeminal small sensory fiber neuropathy in amyotrophic lateral sclerosis. Front Aging Neurosci. 2014;6:278.

37. Silajdžić E, Björkqvist M. A Critical Evaluation of Wet Biomarkers for Huntington's Disease: Current Status and Ways Forward. J Huntingtons Dis. 2018;7(2):109-135. doi: 10.3233/JHD-170273. PMID: 29614689; PMCID: PMC6004896.

38. Roda M, Ciavarella C, Giannaccare G, Versura P. Biomarkers in Tears and Ocular Surface: A Window for Neurodegenerative Diseases. Eye Contact Lens. 2020;46 Suppl 2:S129-S134. doi:10.1097/ICL.0000000000000663 